\title{
Addressing malperfusion first before repairing type A dissection
}

\author{
Yunus Ahmed, MD, Pieter A. J. van Bakel, MD, and Himanshu J. Patel, MD
}

\begin{abstract}
Associate Editor Note-Stanford Type A acute aortic dissection is associated with high operative and overall mortality rates further amplified when a malperfusion syndrome is present. Debate exists as to which component should be addressed first, the dissection or the organ malperfusion. The authors of this invited Expert Opinion provide us with their therapeutic algorithm, which includes percutaneous fenestration of the descending aorta, with or without bare metal stenting of a portion of the abdominal aorta. The central premise of their approach is that the high mortality of fixing the central lesion first is due in part to intensifying the ischemiareperfusion injury to the organs when cardiopulmonary bypass and hypothermic circulatory arrest are added to the mix. There are a few issues with this approach, including concern for increased mortality and/or morbidity with a staged approach, but their institutional data appear to support this approach. Their description of the algorithm, rationale, and outcome data will be useful to surgeons facing the dilemma of malperfusion syndrome in the patient presenting with an acute dissection.
\end{abstract}

\section{Abe DeAnda Jr, MD}

Malperfusion with ischemic end-organ dysfunction (malperfusion syndrome) is seen in up to approximately $30 \%$ of patients presenting with acute type A dissection (TAAD). ${ }^{1}$ This presentation is associated with high morbidity and mortality, and treatment strategies to ensure successful patient survival remain complex. ${ }^{2-4}$ Previous studies have shown malperfusion syndrome, and in particular mesenteric malperfusion, to be an independent predictor of death, with in-hospital mortality rates of up to $63 \% .^{5,6}$ Traditionally, emergent open central aortic repair has been considered the optimal treatment in those patients presenting with malperfusion with end-organ dysfunction following acute TAAD. In a seminal study describing a 25 -year experience

\footnotetext{
From the Department of Cardiac Surgery, University of Michigan Frankel Cardiovascular Center, Ann Arbor, Mich.

H.J.P. was generously supported by the Joe D. Morris Collegiate Professorship, the David Hamilton Fund, and the Phil Jenkins Breakthrough Fund.

Received for publication April 21, 2021; accepted for publication April 21, 2021; available ahead of print May 4, 2021.

Address for reprints: Himanshu J. Patel, MD, Department of Cardiac Surgery, University of Michigan Frankel Cardiovascular Center, 1500 E Medical Center Dr, Ann Arbor, MI 48109 (E-mail: hjpatel@med.umich.edu).

JTCVS Techniques 2021;10:1-5

2666-2507

Copyright (c) 2021 The Author(s). Published by Elsevier Inc. on behalf of The American Association for Thoracic Surgery. This is an open access article under the CC BY-NC-ND license (http://creativecommons.org/licenses/by-nc-nd/4.0/).

https://doi.org/10.1016/j.xjtc.2021.04.029
}

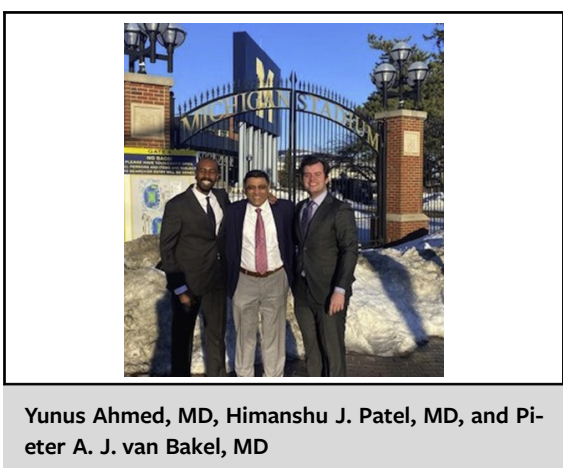

\begin{abstract}
CENTRAL MESSAGE
For acute TAAD patients presenting with malperfusion syndrome, we propose to restore end-organ perfusion first, allow the malperfusion syndrome to resolve, and then repair the proximal aorta.
\end{abstract}

See Commentary on page 6.

of 272 patients, Fann and colleagues ${ }^{7}$ suggested that operative mortality in the setting of visceral malperfusion was $43 \%$.

In 1997, Deeb and colleagues ${ }^{8}$ introduced a novel strategy of operative delay following percutaneous reperfusion with aortic fenestration and subsequent resolution of the malperfusion syndrome. This approach was initiated when outcomes in a series of patients suggested that immediate operative repair in the setting of malperfusion led to mortality from consequences of the inflammatory response from ischemia-reperfusion injury. This injury was sustained when central aortic repair relieved branch vessel obstruction but also included use of deep hypothermic circulatory arrest and prolonged cardiopulmonary bypass. Over recent years, our group 9,10 and others ${ }^{1}$ have suggested acceptable early and late results in patients who survived to operative repair, and these findings supported our novel strategy.

\section{DIAGNOSIS}

Malperfusion can be diagnosed with radiographic findings on computed tomography indicating impaired blood supply to vital organs. Importantly, and in contrast, malperfusion syndrome is defined by the additional 


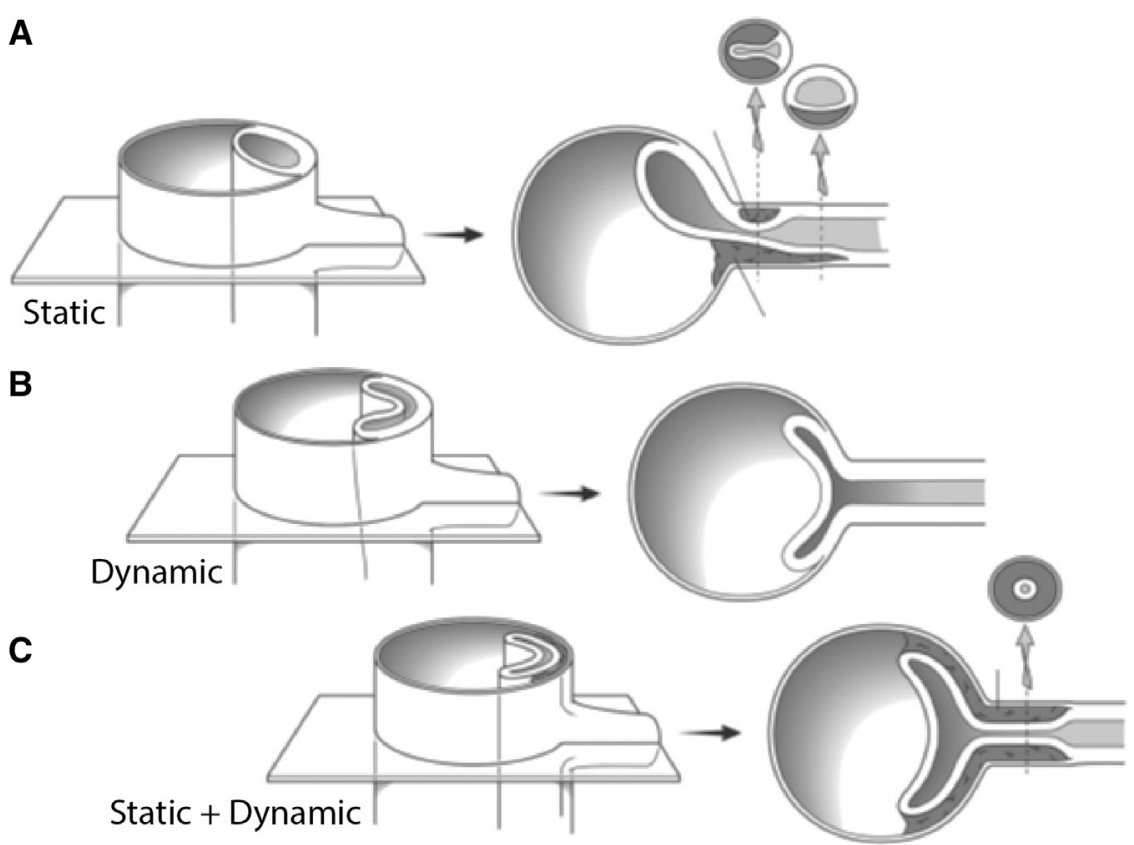

FIGURE 1. Different types of branch vessel obstruction: static (A), dynamic (B), and combination of both (C). Adapted with permission from Williams and colleagues. $^{11}$

presence of ischemic end-organ dysfunction leading to clinical features (abdominal pain, bloody diarrhea, decreased urine output, neurologic deficits) and laboratory findings indicating systemic metabolic abnormalities (elevated creatine, lactate, or liver enzymes). Branch vessel obstruction can result from a (1) static compression, (2) dynamic compression, or (3) a combination of dynamic and static compression (Figure 1, A-C). ${ }^{11}$ Dynamic compression occurs when inadequate or absent re-entry tears cause false lumen pressurization and obstruct a branch vessel emanating from the true lumen by the prolapsing flap over its orifice. Static compression occurs when the dissection flap extends into the branch vessel ostium, and thrombus formation in the false lumen at the orifice results in true lumen obstruction at the branch ostium.

\section{MANAGEMENT STRATEGY}

In assessing patients with TAAD and malperfusion, we must first distinguish between malperfusion and malperfusion syndrome. At our institution, we proposed a patient-specific treatment algorithm ${ }^{10}$ for patients with acute TAAD presenting with malperfusion. Patients presenting with malperfusion alone without evidence of end-organ dysfunction should undergo immediate surgical repair, as should those patients presenting with signs of cardiogenic shock in whom mortality is certain without central aortic repair (Figure 2). ${ }^{10}$ In hemodynamically stable patients with malperfusion syndrome, we choose to fenestrate and stent first to restore end-organ perfusion and delay open aortic repair until after the malperfusion syndrome has resolved. In theory, this reduces the attendant inflammatory response and morbidity from performing complex proximal aortic repair during a time of ischemia-reperfusion injury.

Our method of fenestration has been described and essentially mimics creation of a re-entry tear to decompress the false lumen ${ }^{12,13}$ (Figure 3). ${ }^{14}$ Pressure normalization after fenestration will often restore branch vessel flow in case of a dynamic obstruction. To buttress this flap away from the branch vessel, a bare metal stent is often also placed in the true lumen at the level of the celiac trunk and superior mesenteric artery (Figure 4). Any residual static obstruction may be treated by additional branch vessel stenting. Ultimately, adequate perfusion is confirmed by comparative manometry between the aortic root and the branch vessel itself. Following fenestration, patients are managed with aggressive blood pressure control and supportive care to manage their ischemia-reperfusion injury. Once the malperfusion syndrome is resolved, the patient undergoes an open central aortic repair.

An alternative approach has been proposed by Leshnower and colleagues ${ }^{15}$ from Emory University. They described using thoracic endovascular aortic repair of the descending aorta to expand the true lumen first in patients presenting with acute TAAD complicated by mesenteric malperfusion. Following resolution of end-organ ischemia, these patients then underwent open central aortic repair. We believe the additional risk this may pose is that of false lumen pressurization further in the ascending aorta, theoretically increasing the risk for 


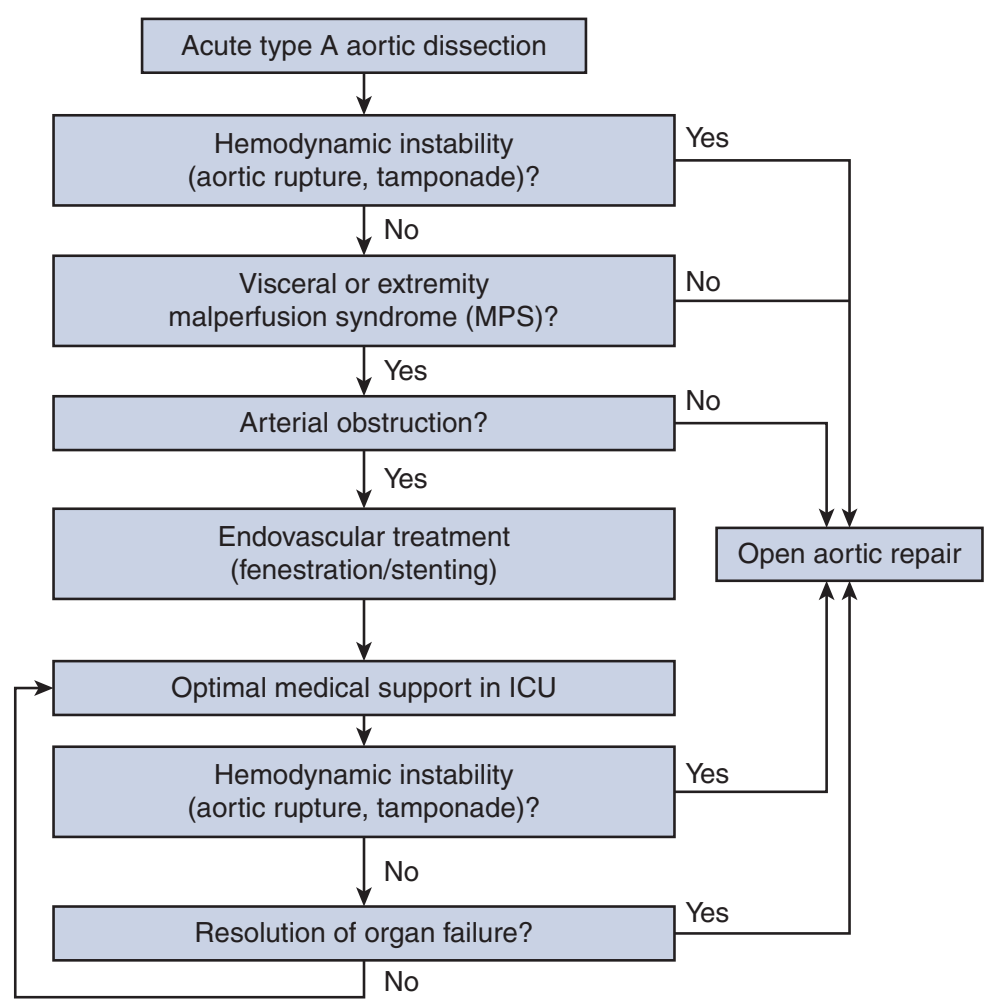

FIGURE 2. Treatment algorithm applied at University of Michigan for clinical decision making in acute type A dissection patients. ICU, Intensive care unit. Reprinted with permission from Yang and colleagues. ${ }^{10}$

rupture. Their patient sample size was likely too small to determine the level of this potential risk.

\section{OUTCOMES}

Our initial study investigating this approach ${ }^{8}$ found that $89 \%$ of patients with TAAD and malperfusion syndrome sustained in-hospital mortality with immediate aortic repair. In those patients in whom central aortic repair was delayed after the endovascular reperfusion strategy, central aortic repair was successful in $85 \%$ and overall survival was $75 \%$. This study suggested that delayed repair was an independent predictor for survival and that patients with malperfusion syndrome who undergo immediate surgery have a 33 times greater likelihood of death.

Lauterbach and colleagues ${ }^{5}$ also proposed a more tailored approach. In their patient cohort of 187 patients, $28 \%$ presented with peripheral malperfusion. They found that following central aortic repair, one-third required additional peripheral intervention. Noting the dismal prognosis in the setting of mesenteric malperfusion, they suggested an algorithm of relief of mesenteric or renal malperfusion and delayed ( 24 hours) central aortic repair.

The inability of central aortic repair alone to restore organ perfusion has been observed in other studies as well. Slonim and colleagues ${ }^{13}$ studied patients presenting with type $A(n=10)$ and type $B(n=30)$ dissection in their description of percutaneous fenestration. They described a high success rate of $92.5 \%$ in restoring end-organ perfusion. Notably, of the 10 patients with type A dissection, $90 \%$ of the fenestration procedures were performed for persistent malperfusion following central aortic repair.

In our first follow-up study of 196 patients, our group ${ }^{9}$ suggested that although our patient-specific approach in patients with TAAD and malperfusion syndrome carries a considerable risk for early mortality, those patients who survive the initial malperfusion have a similar operative mortality $(9.5 \%$ and $8.5 \%$ for uncomplicated TAAD and malperfusion syndrome, respectively) and long-term survival for uncomplicated TAAD compared with patients presenting with complicated TAAD and malperfusion syndrome (mean actuarial survival 95.9 months vs 80.5 months respectively, $P=.45$ ). In this study, of the 70 patients presenting with malperfusion, 47 survived to central aortic repair following a median operative time delay of 4 days. The remaining 23 patients died either as a consequence of complications from the malperfusion syndrome $(\mathrm{n}=11)$ or from aortic rupture while awaiting resolution of the malperfusion $(\mathrm{n}=12)$.

Several groups have continued to advocate for immediate primary central aortic repair in the setting of malperfusion. Geirsson and colleagues ${ }^{1}$ from the University of 


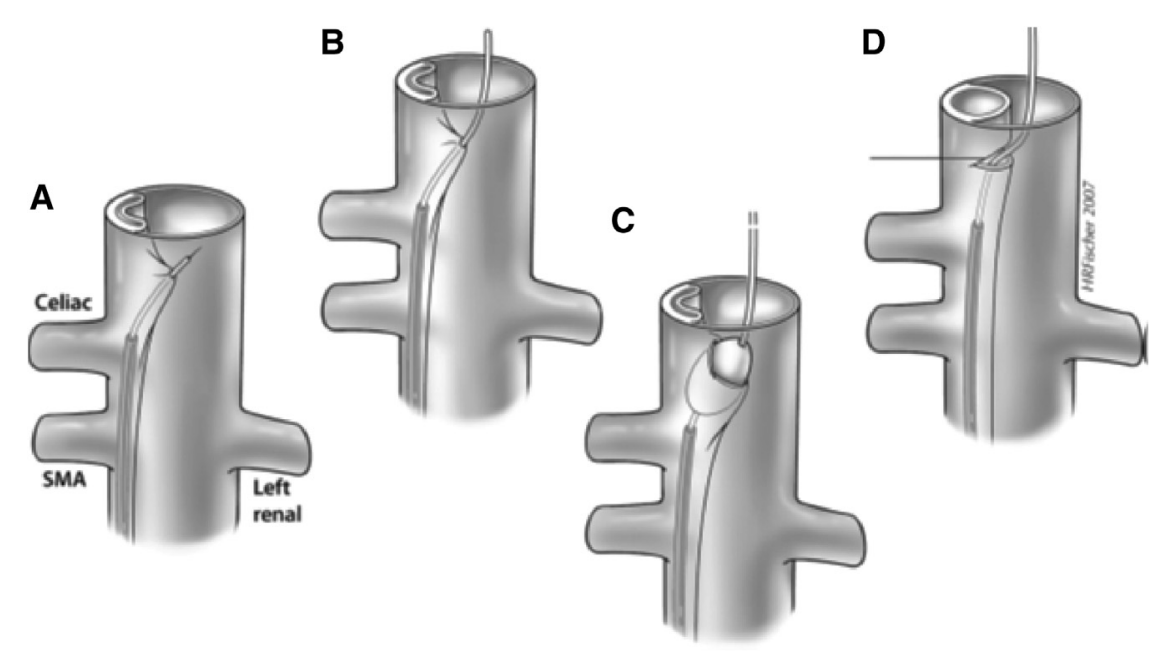

FIGURE 3. Creating of a fenestration to equalize pressure gradients across lumens. The directection of the needle puncture is determined using intravascular ultrasound at the level of the needle tip, generally from true lumen into the false lumen (A). The catheter is advanced of the sylet into the false lumen, after which a wire is placed into the false lumen (B). An angioplasty balloon is centered across the flap and inflated to widen the fenestration and promote flow from the false-to-true lumen (C), thus creating a fenestration (D). SMA, Superior mesenteric artery. Reprinted with premission from Khayat and colleagues. ${ }^{14}$

Pennsylvania found that although early central aortic repair in patients with acute TAAD presenting with malperfusion was associated with increased in-hospital mortality, long-term survival may be similar between these groups in all but those presenting with cerebral malperfusion. In 2018, Chiu and colleauges ${ }^{16}$ from Stanford suggested that both in-hospital mortality and late survival were similar between those presenting with or without malperfusion. The major difference between groups in this study was that of an increased incidence of branch vessel intervention at 10 years $(12.5 \%$ vs $5.7 \%$, respectively). On the basis of their results, the authors advocated for immediate aortic repair in all patients. Although these results may suggest similar outcomes to our strategy, the patient profile in their cohort was different; in particular, the incidence of

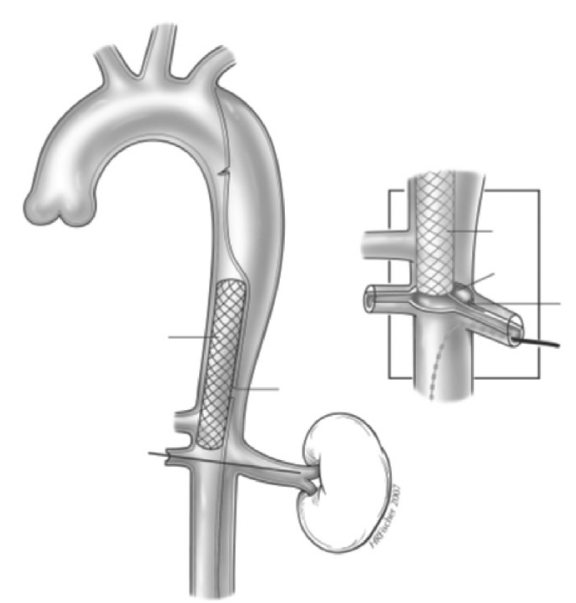

FIGURE 4. True lumen stenting to ensure vessel patency. Reprinted with premission from Khayat and colleagues. ${ }^{14}$ mesenteric malperfusion was lower, which is known to be associated with lower survival.

Our most recent work regarding this strategy reported our 20-year experience ${ }^{10}$ in 597 patients (135 with malperfusion syndrome) and contrasted our experience between the first and second decades of this operative delay strategy. Operative mortality improved $(21.0 \%$ vs $10.7 \%$, $P<.001)$ and mortality from aortic rupture decreased $(16 \%-4 \%, P=.05)$, likely due to multiple reasons. In total, $87 \%$ of the mortality was attributable to organ failure in those dying before aortic repair. The median delay to surgery in the second decade was now down to 2 days as a result of delaying only until assessment suggested an ability to tolerate surgery, rather than waiting until organ function completely recovered. These findings supported our staged approach.

Mesenteric malperfusion in particular has been identified as a major risk factor for mortality in TAAD. Several multicenter studies have described dismal results with management of TAAD with mesenteric malperfusion, including the International Registry of Acute Aortic Dissection, and the Emilia Romagna Cardiac Surgery Registry. In our recent work focusing on this problem, we described an in-hospital mortality rate of $39 \%$ for all patients presenting with mesenteric malperfusion $(\mathrm{n}=82) .{ }^{17}$ In addition, more than $50 \%$ of presenting patients needed superior mesenteric artery stents, focal thrombolysis, and/or suction thromboembolectomy, suggesting that upfront central aortic repair might have only been successful at relieving malperfusion in a subset of this group. Finally, futile central aortic repair was avoided in those 20 patients $(24 \%)$ who died from organ failure. 
Two particular items deserve special final mention as a source of confusion in the literature. ${ }^{18}$ The first area to note is the variability in the number of vascular beds affected by malperfusion reported in studies of timing of surgery. Whereas significant end-organ dysfunction may only be present in one vascular bed, our approach with inclusion of manometric assessment of each territory suggests that more frequently, multiple beds are involved. Other investigators have suggested that the number of vascular territories affected directly correlates with increasing mortality. ${ }^{2-4}$ Second, different studies have differing rates of celiac and mesenteric malperfusion relative to other beds. With the strong association of malperfusion of this particular bed and early mortality, this variability may also affect outcomes reported between studies and render comparison much more difficult.

\section{CONCLUSIONS}

While immediate open central aortic repair remains the treatment of choice in the vast majority of patients with TAAD, when treating those patients presenting with ischemic end-organ dysfunction as a consequence of sustained malperfusion, we propose an algorithm to percutaneously reperfuse first, resolve the malperfusion syndrome, and then repair the proximal aorta. While there is still risk of aortic rupture, futile attempts to perform aortic repair in this setting are avoided. With increasing experience, there appears to be a marked reduction in risk of aortic rupture. In particular, with this strategy, shortand long-term survival among patients treated with this approach and undergoing operation has shown to be similar to those patients presenting with acute TAAD without evidence of ischemic end-organ dysfunction.

\section{Conflict of Interest Statement}

H.J.P. has consulting agreements with W. L. Gore \& Associates, Inc, and Medtronic. All other authors reported no conflicts of interest.

The Journal policy requires editors and reviewers to disclose conflicts of interest and to decline handling or reviewing manuscripts for which they may have a conflict of interest. The editors and reviewers of this article have no conflicts of interest.

\section{References}

1. Geirsson A, Szeto WY, Pochettino A, McGarvey ML, Keane MG, Woo YJ, et al. Significance of malperfusion syndromes prior to contemporary surgical repair for acute type A dissection: outcomes and need for additional revascularizations. Eur J Cardiothorac Surg. 2007:32:255-62.

2. Girdauskas E, Kuntze T, Borger MA, Falk V, Mohr FW. Surgical risk of preoperative malperfusion in acute type A aortic dissection. J Thorac Cardiovasc Surg. 2009; 138:1363-9.

3. Czerny M, Schoenhoff F, Etz C, Englberger L, Khaladj N, Zierer A, et al. The impact of pre-operative malperfusion on outcome in acute type a aortic dissection: results from the GERAADA Registry. J Am Coll Cardiol. 2015;65: 2628-35.

4. Pacini D, Leone A, Belotti LMB, Fortuna D, Gabbieri D, Zussa C, et al. Acute type A aortic dissection: sgnificance of multiorgan malperfusion. Eur $J$ Cardiothorac Surg. 2013;43:820-6.

5. Lauterbach SR, Cambria RP, Brewster DC, Gertler JP, Lamuraglia GM, Isselbacher EM, et al. Contemporary management of aortic branch compromise resulting from acute aortic dissection. J Vasc Surg. 2001;33: $1185-92$.

6. Di Eusanio M, Trimarchi S, Patel HJ, Hutchinson S, Suzuki T, Peterson MD, et al. Clinical presentation, management, and short-term outcome of patients with type A acute dissection complicated by mesenteric malperfusion: observations from the International Registry of Acute Aortic Dissection. J Thorac Cardiovasc Surg. 2013;145:385-90.e1.

7. Fann JI, Sarris GE, Mitchell RS, Shumway NE, Stinson EB, Oyer PE, et al. Treatment of patients with aortic dissection presenting with peripheral vascular complications. Ann Surg. 1990;212:705-13.

8. Deeb GM, Williams DM, Boiling SF, Quint LE, Monaghan H, Sievers J, et al. Surgical delay for acute type A dissection with malperfusion. Ann Thorac Surg. 1997;64:1669-77.

9. Patel HJ, Williams DM, Dasika NL, Suzuki Y, Deeb GM. Operative delay for peripheral malperfusion syndrome in acute type A aortic dissection: a long-term analysis. J Thorac Cardiovasc Surg. 2008;135:1288-96.

10. Yang B, Rosati CM, Norton EL, Kim KM, Khaja MS, Dasika N, et al. Endovascular fenestration/stenting first followed by delayed open aortic repair for acute type A aortic dissection with malperfusion syndrome. Circulation. 2018;138: 2091-103.

11. Williams DM, Lee DY, Hamilton BH, Marx MV, Narasimham DL, Kazanjian SN, et al. The dissected aorta: part III. Anatomy and radiologic diagnosis of branch-vessel compromise. Radiology. 1997;203:37-44.

12. Williams DM, Andrews JC, Marx MV, Dasika NL, Kazanjian SN, Prince MR et al. Creation of reentry tears in aortic dissection by means of percutaneous balloon fenestration: gross anatomic and histologic considerations. J Vasc Interv Radiol. 1993:4:75-83.

13. Slonim SM, Miller DC, Mitchell RS, Semba CP, Razavi MK, Dake MD. Percutaneous balloon fenestration and stenting for life-threatening ischemic complications in patients with acute aortic dissection. J Thorac Cardiovasc Surg. 1999 117:1118-27.

14. Khayat M, Cooper KJ, Khaja MS, Gandhi R, Bryce YC, Williams DM. Endovascular management of acute aortic dissection. Cardiovasc Diagn Ther. 2018; 8(suppl 1):S97-107.

15. Leshnower BG, Keeling WB, Duwayri YM, Jordan WD Jr, Chen EP. The "thoracic endovascular aortic repair-first" strategy for acute type A dissection with mesenteric malperfusion: initial results compared with conventional algorithms. J Thorac Cardiovasc Surg. 2019;158:1516-24.

16. Chiu P, Tsou S, Goldstone AB, Louie M, Woo YJ, Fischbein MP. Immediate operation for acute type A aortic dissection complicated by visceral or peripheral malperfusion. J Thorac Cardiovasc Surg. 2018;156:18-24.e3.

17. Yang B, Norton EL, Rosati CM, Wu X, Kim KM, Khaja MS, et al Managing patients with acute type A aortic dissection and mesenteric malperfusion syndrome: a 20-year experience. J Thorac Cardiovasc Surg. 2019;158:675-87.e4.

18. Patel HJ. Peas and carrots, apples and oranges: not all malperfusion is the same. $J$ Thorac Cardiovasc Surg. 2018;156:25-6.

Key Words: aortic dissection, aortic repair 